\title{
Costs and Cost Drivers Associated with Non- Small-Cell Lung Cancer Patients Who Received Two or More Lines of Therapy in Europe
}

This article was published in the following Dove Press journal: ClinicoEconomics and Outcomes Research

\author{
Katharina Verleger' \\ John R Penrod ${ }^{2}$ \\ Melinda Manley Daumont ${ }^{3}$ \\ Caitlyn Solem (1D) \\ Linlin Luo ${ }^{4}$ \\ Cynthia Macahilig 5 \\ Nadine Hertel ${ }^{6}$ \\ 'Pharmerit International, Berlin, \\ Germany; ${ }^{2}$ Bristol-Myers Squibb, \\ Princeton, NJ, USA; ${ }^{3}$ Bristol-Myers \\ Squibb, Braine-L’alleud, Belgium; \\ ${ }^{4}$ Pharmerit International, Bethesda, MD, \\ USA; ${ }^{5}$ Medical Data Analytics, Parsippany, \\ NJ, USA; ' ${ }^{6}$ Bristol-Myers Squibb, \\ Uxbridge, UK
}

Purpose: Advanced non-small-cell lung cancer (aNSCLC; stage IIIB/IV) presents a substantial clinical burden to society; reliable estimates of its economic burden are lacking. Therefore, this study aimed to quantify real-world health care resource utilization (HCRU) and costs of patients with squamous (SQ) and non-SQ (NSQ) aNSCLC who received two or more lines of treatment $(2 \mathrm{~L}+)$ in Europe, and to describe cost-predictors.

Methods: The LENS (Leading the Evaluation of Non-squamous and Squamous NSCLC) retrospective chart review study collected data from $2 \mathrm{~L}+$ patients with aNSCLC diagnosed between 07/2009 and 08/2011 (wave 1) or 07/2010 and 09/2012 (wave 2) in France, Germany, Italy, Spain, England, the Netherlands, and Sweden. Patients were followed from diagnosis through most recent visit/death. A weighted average of countryspecific unit costs (2018 Euro) was applied to systemic anti-cancer therapy usage and HCRU (hospital/emergency department visit, surgery, radiotherapy, ancillary care, biomarker testing) to determine the total cost from aNSCLC diagnosis to death. Generalized linear models (gamma distribution, log link) were used to assess clinical and demographic predictors.

Results: Of 973 2L + aNSCLC patients, median overall survival (OS) was 1.5 years from advanced diagnosis (range: 0.2-5.3; median OS: 1.4 [SQ], 1.6 [NSQ]), 79.0\% died during follow-up. Weighted mean total per-patient costs were $€ 21,273$, ranging from $€ 17,761$ (England) to $€ 30,854$ (Sweden), and $€ 15,446$ (SQ) to $€ 26,477$ (NSQ). Systemic drug costs comprised $77.4 \%$ of total costs. Insurance status, presence of epidermal growth factor receptor $(E G F R)$ mutation, SQ histology, age, alcohol abuse, and year of diagnosis were significant predictors for lower total costs per patient-month, Eastern Cooperative Oncology Group performance status (ECOG PS) $\geq 1$ and country for higher costs.

Conclusion: In the era pre-immunotherapy, HCRU and costs were substantial in aNSCLC $2 \mathrm{~L}+$ patients, with most of the costs accrued prior to start of 2L. NSQ patients incurred significantly higher total costs than SQ patients in all participating countries.

Keywords: carcinoma, non-small-cell lung, observational study, cost of illness, health care costs

\section{Introduction}

Lung cancer is the most common cancer in the world and the leading cause of cancer deaths among people over 65 , with a three-fold elevated risk for men compared to women. ${ }^{1}$ In $2018,183,100$ persons were predicted to die of lung cancer in the European Union. $^{2}$ Non-small-cell lung cancer (NSCLC) accounts for $85 \%$ to $90 \%$ of all lung cancer diagnoses. ${ }^{3,4}$ Survival rates are poor, ${ }^{5}$ particularly among the majority of patients who present with advanced NSCLC (aNSCLC), ie, stages IIIB or IV of the International
Correspondence: Katharina Verleger Pharmerit International, Krausenstr 8 Berlin I0II7, Germany

Tel +493030807024

Email kverleger@pharmerit.com 
Staging Committee of the International Association for the Study of Lung Cancer (ISC-IASLC). ${ }^{6}$

Although the clinical burden of the disease is well understood, reliable estimates of the economic burden of aNSCLC to society in Europe are lacking since the cost estimates are not based on detailed real-world evidence (RWE). ${ }^{7}$ Consequently, the direct costs incurred by aNSCLC are unknown, and information on the drivers of costs is scarce. The LENS study (Leading the Evaluation of Non-squamous and Squamous NSCLC) aimed to quantify real-world health care resource utilization (HCRU) related to aNSCLC and their associated costs of patients with squamous (SQ) and non-squamous (NSQ) aNSCLC who received two or more lines of treatment $(2 \mathrm{~L}+)$ in seven European countries. In addition, this analysis aimed to describe predictors of real-world costs per patient.

\section{Methods}

\section{Data Source}

Data for this retrospective, observational patient chart review study were sampled by medical oncologists, hematologists/oncologists, and pulmonologists, who were recruited from national physician databases in England, France, Germany, Italy, the Netherlands, Spain, and Sweden. Participating physicians had to be practicing oncologists or pulmonologists, spend at least $50 \%$ of their practice time treating patients with aNSCLC, and be willing to obtain all data of interest. Participating physicians were responsible for patient selection, data abstraction, and completion of the patient case report forms retrospectively following explicit study instructions.

Due to the retrospective nature of the study, an Ethics Committee/Institutional Review Board application for exemption or waiver was obtained. All participating physicians and patients remained anonymous to the sponsor, and all data were de-identified. All data were collected and processed with adequate precautions to ensure confidentially and compliance with applicable data privacy protection laws and regulations at the time of data collection.

Real-world medical and treatment data were extracted from patient charts during the follow-up period from baseline (diagnosis of aNSCLC) to the most recent physician visit, known condition, or death. In addition, HCRU (ie, aNSCLC-related hospital/emergency department [ED] visit, surgery, radiotherapy, ancillary care [hospice, nursing home, in-home care], biomarker test) and systemic anti-cancer treatment including doses were extracted. Data were obtained in two consecutive waves: Wave 1 was conducted from 08/2013 to 04/2015 in France, Germany, Italy, and Spain; Wave 2 from 04/2015 to $01 / 2017$ in England, the Netherlands, and Sweden.

\section{Patient Selection}

Adult 2L+ patients with aNSCLC diagnosed from 07/2009 through/to 08/2011 (wave 1) or 07/2010 through/to 09/ 2012 (wave 2) were eligible for inclusion. The patient's medical chart was required to provide all data on age, sex, date of aNSCLC diagnosis, all lines of treatment (including agent types and dosage), therapy response, date of disease progression, date of last follow-up, and vital status. Patients who were enrolled in a cancer treatment-related clinical trial since date of aNSCLC diagnosis were excluded. Target enrollment was 200 patients per country, with a proportionate divide of SQ and NSQ patients.

\section{Costing}

Country-specific unit costs were obtained from publicly available databases and adjusted for inflation to 2018 Euro $(€)$. To adjust for country-specific unit cost differences, all cost analyses in this manuscript were computed using the following method: an averageweighted cost per unit was calculated, based on number of patients within the total cohort per country (Weighted unit cost $=\sum$ Unit cost $\mathrm{Country} * \mathrm{~N}_{\text {Country }} / \mathrm{N}_{\text {Overall }}$ ). If a unit cost was unavailable in a country, a unit cost from another country was used as a proxy. The weighted average unit costs were then multiplied by the amount of systemic anti-cancer drug usage and HCRU units to derive the total aNSCLC-related costs from aNSCLC diagnosis to death overall as well as per country. A table of weighted unit costs, and of unit costs by country including proxy assumptions with source data is available in the Supplementary Materials.

\section{Statistical Analyses}

Numerical variables were described by median and $95 \%$ confidence interval (CI) or by mean and standard deviation (SD). Categorical variables were described by frequencies and percentages. Chi-squared, Fisher's exact, and Student's t-tests were used to determine significant differences between groups using a threshold of $p<0.05$ for significance, unless otherwise stated. HCRU was 
calculated and costs were estimated per patient as well as per patient-month during four time periods: "Total costs" from aNSCLC diagnosis to death or last date of followup; "Pre-progression" from aNSCLC diagnosis to first progression or start of second-line (2L) treatment; "Postprogression" from first progression or start of $2 \mathrm{~L}$ treatment to last date of follow-up or (if patient died) last month of life (end of life, EOL); and "EOL," 28 days prior to a patient's death. A patient-month was defined as the mean length of observation time in months (sum of total observation time in days divided by 28 days, calculated per patient). Time-to-event results were derived from Kaplan-Meier survival analysis.

Prognostic factors associated with costs from aNSCLC diagnosis (total costs per patient, and costs per patient-month) were examined through generalized linear models (GLMs) with log link and gamma distribution used; clinically relevant and independent variables tested for inclusion are provided in Table 1. In order to select a model that balanced the clinical and statistical importance of different potential covariates, the GLMs selection process for the outcome total weighted costs was as follows:
1. In the first series of analyses (step 1), candidate variables were entered individually to understand bivariate statistical associations.

2. Step 2 included clinically relevant variables, which were identified a priori within the statistical analysis plan; these were variables that the study team considered important to include in models regardless of statistical significance.

3. Step 3 applied a backward selection process to select variables for inclusion, using only variables from step 1 that had $\mathrm{p}<0.1$.

4. The final model (step 4) included all clinically relevant variables regardless of statistical significance, plus the significant variables from step 3 .

To account for varying patient follow-up time and overall survival (OS), the final model was then also used to explore prognostic factors and their odds ratios associated with costs per patient-month.

As a sensitivity analysis, the cost analyses were conducted in a subset of patients with complete follow-up data until death.

Data analysis was conducted using SAS software, version 9.4 of the SAS System for Windows (Cary, NC, USA).

Table I Model Development and Input Variables

\begin{tabular}{|c|c|c|c|c|c|c|}
\hline \multirow[t]{2}{*}{ Variable } & \multirow[t]{2}{*}{ Reference Category } & \multirow[t]{2}{*}{ Comparison Categories } & \multicolumn{4}{|c|}{ Model $^{\mathbf{a}}$} \\
\hline & & & $\mathbf{I}$ & 2 & 3 & 4 \\
\hline Age, years & $<65$ & $65-79 ;>79$ & $x$ & $x$ & $x$ & $x$ \\
\hline Sex & Female & Male & $x$ & $x$ & $x$ & $x$ \\
\hline Country & Germany & Spain; France; Italy; Netherlands; Sweden; England & $x$ & $x$ & $x$ & $x$ \\
\hline Histology & Non-squamous & Squamous & $x$ & $x$ & $x$ & $x$ \\
\hline ISC-IASLC disease stage & IIIIB & IV & $x$ & $x$ & & $x$ \\
\hline ECOG PS score & 0 & I; $\geq 2$; Unknown & $x$ & $x$ & & $x$ \\
\hline Smoking status & Never & Former; Current & $x$ & $x$ & $x$ & $x$ \\
\hline Alcohol abuse & No; Unknown & Yes & $x$ & $x$ & $x$ & $x$ \\
\hline Year of diagnosis & 2009 & $2010 ; 2011 ; 2012$ & $x$ & $x$ & $x$ & $x$ \\
\hline Private-based practice & No & Yes & $x$ & & $x$ & \\
\hline Insurance & Private & Public; Unknown & $x$ & & $x$ & $x$ \\
\hline Weight loss & No & Yes & $x$ & & & \\
\hline Metastasis present & No & Yes & $x$ & & & \\
\hline Malignant pleural effusion & No & Yes & $x$ & & & \\
\hline \multicolumn{7}{|l|}{ Presence of biomarker } \\
\hline EGFR mutation & Absent; Not tested; Inconclusive & Present & $x$ & & $x$ & $x$ \\
\hline ALK translocation & Absent; Not tested; Inconclusive & Present & $x$ & & & \\
\hline KRAS mutation & Absent; Not tested; Inconclusive & Present & $x$ & & & \\
\hline
\end{tabular}

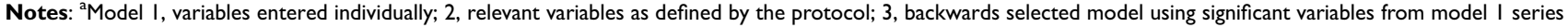
$(\mathrm{p}<0.1) ; 4$, significant variables from model 3 plus clinically relevant variables (final model).

Abbreviations: ALK, anaplastic lymphoma receptor tyrosine-kinase gene; ECOG PS, Eastern Cooperative Oncology Group performance status; EGFR, epidermal growth factor receptor gene; ISC-IASLC, International Staging Committee of the International Association for the Study of Lung Cancer; KRAS, Kirsten rat sarcoma viral oncogene. 


\section{Results}

\section{Baseline Characteristics}

The analysis included data from $9732 \mathrm{~L}+$ patients with aNSCLC from 193 sites across the seven European countries, who were followed for a mean of 1.7 years (range: $0.2-5.3$ years), until their most recent visit $(21.0 \%)$ or death $(79.0 \%)$. The 769 patients who died constitute the sensitivity analysis set. Due to the sampling strategy, a similar number of SQ and NSQ histology patients were included in the overall cohort.

Most patients were male (71.0\%), had ISC-IASLC disease stage IV at advanced diagnosis (81.5\%), and Eastern Cooperative Oncology Group performance status (ECOG PS) of 1 (60.4\%); the mean age was 64.3 years at diagnosis (Table 2). Data analyzed by histology revealed that patients with SQ aNSCLC were generally older (65.0 vs 63.7 years) and more likely to be male than patients with NSQ aNSCLC (80.2\% vs $62.7 \%)$. Disease stage and ECOG PS were similarly distributed across patients with SQ and NSQ histology. When the data were analyzed by country it was observed that the percentage of patients with an ECOG PS $\geq 2$ was at $22.7 \%$ higher in Germany than in all other countries. Most patients (80.2\%) with NSQ disease were tested for an epidermal growth factor receptor $(E G F R)$ mutation, compared with $26.2 \%$ of patients with SQ disease. Presence of EGFR mutation was also higher in patients with NSQ disease than in patients with SQ aNSCLC (31.1\% vs $10.2 \%)$. The percentage of patients who received a biomarker test and tested positively for the presence of EGFR mutation ranged from $10.3 \%$ in Sweden to $28.2 \%$ in Italy. On average, patients at diagnosis were oldest in Sweden (67.5 years) and youngest in England (60.9 years). The proportion of female patients ranged from $23.6 \%$ in Italy to $46.2 \%$ in England; the share of SQ patients ranged from $13.8 \%$ in Sweden to $53.8 \%$ in Germany.

Median OS from aNSCLC diagnosis was 1.5 years (range: $0.2-5.3$ years) for the overall population, 1.4 years for SQ patients (range: 0.2-5.3 years), and 1.6 years for NSQ patients (range: 0.3-4.8 years). Among SQ patients, median OS was highest in Italy (1.8 years), followed by Germany and England (1.5 years), Sweden (1.3 years), Spain (1.2 years), and France and the Netherlands (1.1 years). Among NSQ patients, median OS was highest in Germany (1.9 years), Sweden, Spain, and Italy (all 1.7 years), and France, the Netherlands, and England (1.2 years)

\section{Health Care Resource Utilization}

Over the patients' follow-up period, ie from diagnosis through most recent visit/death, $16.1 \%$ of patients were hospitalized at least once and $8.6 \%$ required one or more ED visits (Table 3). The distribution of HCRU differed significantly between countries, ranging from $7.7 \%$ of patients with at least one hospital admission in Italy to $51.7 \%$ in Sweden $(p<0.001)$. Among patients who were hospitalized, median length of hospitalization was 0.8 days per patient-month (range: $<0.1$ to 4.5 days). Hospitalizations and ED visits occurred most frequently once patients had progressed/were receiving $2 \mathrm{~L}$ treatment until EOL.

During the patients' follow-up period, $20.7 \%$ had received radiotherapy and $3.8 \%$ had undergone surgery. Within this cohort, biomarker testing was common, with $49.6 \%$ of patients tested for at least one biomarker. Referrals to other specialists happened most often in the period between aNSCLC diagnosis and first progression/ start of $2 \mathrm{~L}$. In addition to the above-described resource utilization, $18.4 \%$ of patients had received ancillary care with a mean duration of 2.2 days (SD: 1.8 days) per patient-month. In Sweden, $27.5 \%$ of patients utilized hospice care with a mean duration of 2.7 days (SD: 1.6 days) per patient-month, followed by $15.7 \%$ in France with 2.2 days (SD: 1.3 days). The majority of ancillary care was used during EOL.

\section{Systemic Anti-Cancer Drug Usage}

As per study design, all patients received two or more lines of systemic treatment; $13.6 \%$ received third-line treatment. Consistent with 2016 guidelines by the European Society for Medical Oncology, ${ }^{8}$ platinum-based doublet chemotherapy was the most common first-line (1L) treatment and docetaxel, the most common $2 \mathrm{~L}$ treatment in all countries.

\section{Costs Related to aNSCLC and Cost Drivers}

Having multiplied the systemic anti-cancer drug usage and HCRU units by the weighted average unit costs across countries, the mean per-patient costs related to aNSCLC over the follow-up period for $2 \mathrm{~L}+$ patients amounted to $€ 21,273$ (SD: $€ 34,981$; median: $€ 13,235$; Figure 1) or $€ 1,293$ per patient-month. Mean per-patient total costs were lowest in England ( $€ 17,761$; SD: $€ 11,135$; median: $€ 15,723$; mean per patient-month: $€ 1,169)$ and highest in Sweden (mean: $€ 30,854$; SD: $€ 21,443$; median: $€ 28,254$; 


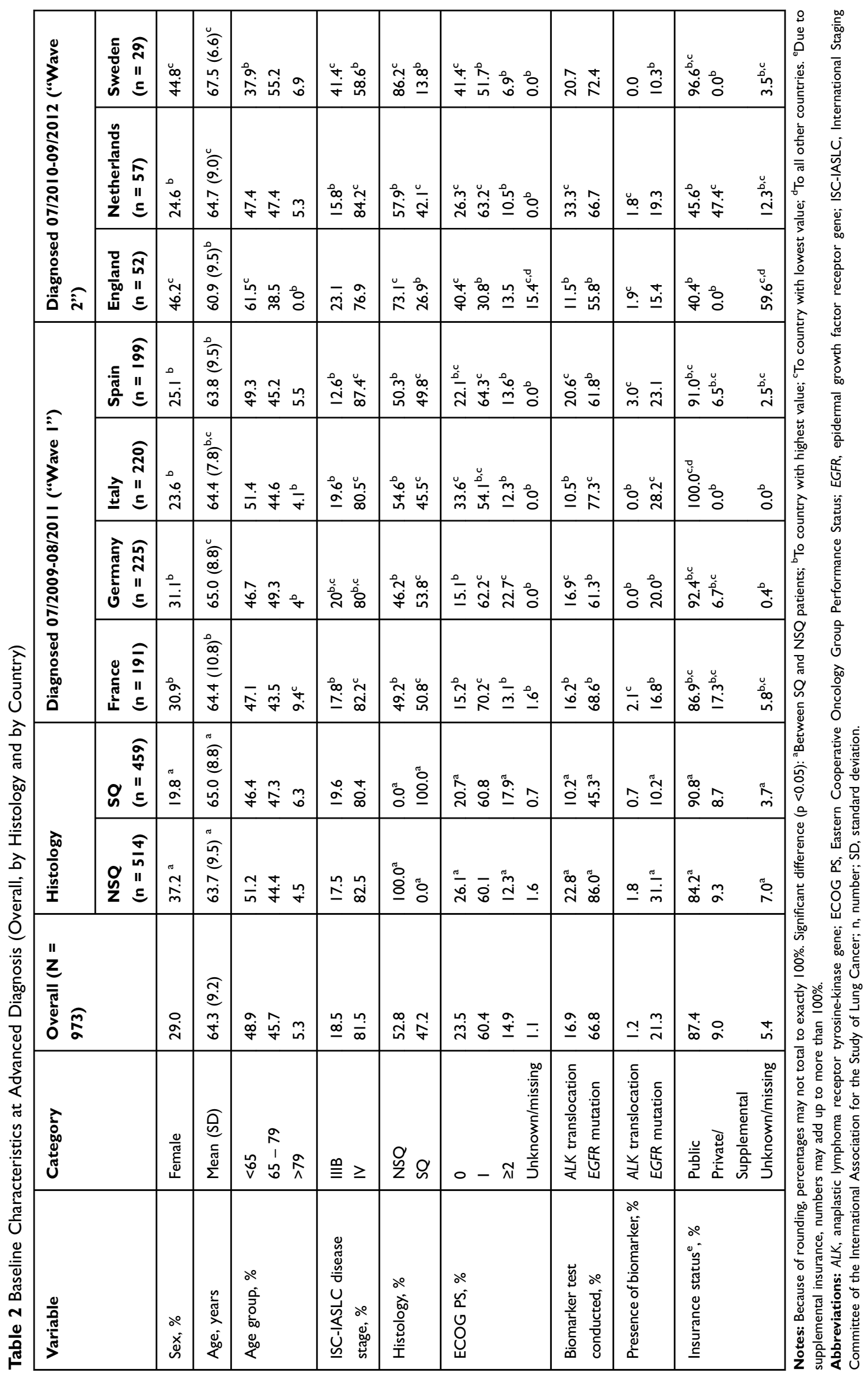


Table 3 aNSCLC-Related Health Care Resource Utilization per 2L+ Patient (Overall, by Histology and by Country)

\begin{tabular}{|c|c|c|c|c|c|c|c|c|c|c|}
\hline \multirow[t]{3}{*}{ Resource } & \multicolumn{10}{|c|}{ No. (\%) of Patients } \\
\hline & \multirow{2}{*}{$\begin{array}{l}\text { Overall } \\
(N=973)\end{array}$} & \multicolumn{2}{|c|}{ By Histology } & \multicolumn{7}{|c|}{ By Country } \\
\hline & & $\begin{array}{l}\text { NSQ } \\
(n=514)\end{array}$ & $\begin{array}{l}\text { SQ } \\
(n=459)\end{array}$ & $\begin{array}{l}\text { France } \\
(n=191)\end{array}$ & $\begin{array}{l}\text { Germany } \\
(n=225)\end{array}$ & $\begin{array}{l}\text { Italy } \\
(n=220)\end{array}$ & $\begin{array}{l}\text { Spain } \\
(n=199)\end{array}$ & $\begin{array}{l}\text { England } \\
(n=52)\end{array}$ & $\begin{array}{l}\text { Netherlands } \\
(n=57)\end{array}$ & $\begin{array}{l}\text { Sweden } \\
(n=29)\end{array}$ \\
\hline Hospitalization & $157(16.1)$ & $90(17.5)$ & $67(14.6)$ & $15(7.9)$ & $24(10.7)$ & $17(7.7)$ & $40(20.1)$ & $26(50.0)$ & $20(35.1)$ & $15(51.7)$ \\
\hline ED visit & $84(8.6)$ & $49(9.5)$ & $35(7.6)$ & $8(4.2)$ & $16(7.1)$ & $7(3.2)$ & $18(9)$ & $12(23.1)$ & $17(29.8)$ & $6(20.7)$ \\
\hline Biomarker testing & $483(49.6)$ & $335(65.2)$ & 148 (32.2) & $114(59.7)$ & $99(44.0)$ & $109(49.5)$ & $90(45.2)$ & $24(46.2)$ & $31(54.4)$ & $16(55.2)$ \\
\hline $\begin{array}{l}\text { Diagnostic } \\
\text { procedures }^{\mathrm{a}}\end{array}$ & $591(60.7)$ & $307(59.7)$ & $284(61.9)$ & 151 (79.1) & $158(70.2)$ & $123(55.9)$ & $135(67.8)$ & $0(0)$ & $12(21.1)$ & $12(4 I .4)$ \\
\hline Surgery & $37(3.8)$ & $15(2.9)$ & $22(4.8)$ & II (5.8) & $22(9.8)$ & $I(0.5)$ & $I(0.5)$ & $2(3.8)$ & $0(0)$ & $0(0)$ \\
\hline Radiotherapy & $20 \mathrm{I}(20.7)$ & $112(21.8)$ & $89(19.4)$ & $38(19.9)$ & $29(12.9)$ & $33(15)$ & $40(20.1)$ & $26(50.0)$ & $20(35.1)$ & $15(51.7)$ \\
\hline Referrals & $81(8.3)$ & $55(10.7)$ & $26(5.7)$ & $10(5.2)$ & $10(4.4)$ & $14(6.4)$ & $4(2.0)$ & $24(46.2)$ & $16(28.1)$ & $3(10.3)$ \\
\hline Ancillary services ${ }^{\mathrm{b}}$ & $179(18.4)$ & $95(18.5)$ & $84(18.3)$ & $33(17.3)$ & $30(13.3)$ & $56(25.5)$ & $28(14.1)$ & $9(17.3)$ & $10(17.5)$ & $13(44.8)$ \\
\hline
\end{tabular}

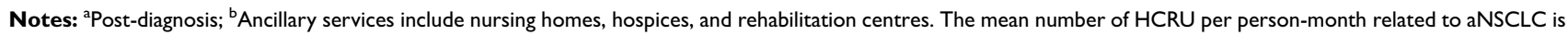
provided in the Supplementary Materials.

Abbreviations: $2 \mathrm{~L}+$, two or more lines of treatment; aNSCLC, advanced non-small-cell lung cancer; ED, emergency department; NSQ, non-squamous; SQ, squamous.

mean per patient-month: €1447). The histology-specific analysis showed that mean total costs were substantially lower for SQ patients $(€ 15,446$; SD: $€ 25,810$; median: $€ 8,748$; mean per patient-month: €915) compared to NSQ patients (mean: €26,477; SD: €40,820; median: $€ 19,604$; mean per patient-month: $€ 1631)$. Costs for systemic anti-cancer medication accounted for $77.4 \%$ of mean costs per patient (SQ: 74.1\%; NSQ: 79.1\%), followed by ancillary care $(7.6 \%$ of total mean costs), radiotherapy $(7.0 \%)$, and hospitalizations (4.5\%) as the next largest cost contributors (Figure 2). Similar to the total costs, costs were much lower in SQ patients than in NSQ patients both pre- and post-progression. During EOL, costs were similar between histologies (Figure 3). SQ patients accrued $52.1 \%$ of total mean costs preprogression, $38.9 \%$ post-progression (prior to start of 2L), and 9.0\% during EOL. NSQ patients accrued 54.8\% pre-progression, $38.2 \%$ post-progression, and $7.0 \%$ during EOL.

While ISC-IASLC stage and ECOG PS were not significant predictors of total costs from aNSCLC diagnosis to most recent visit/death in the bivariate analyses (step 1), they were still included in the final model (step 4). In this final multivariable model, SQ histology, public insurance status, advanced age (age categories "65-79 years" and ">79 years"), presence of EGFR mutation, a history of alcohol abuse, and being diagnosed in 2010, predicted significantly lower total costs per patient (Figure 4).

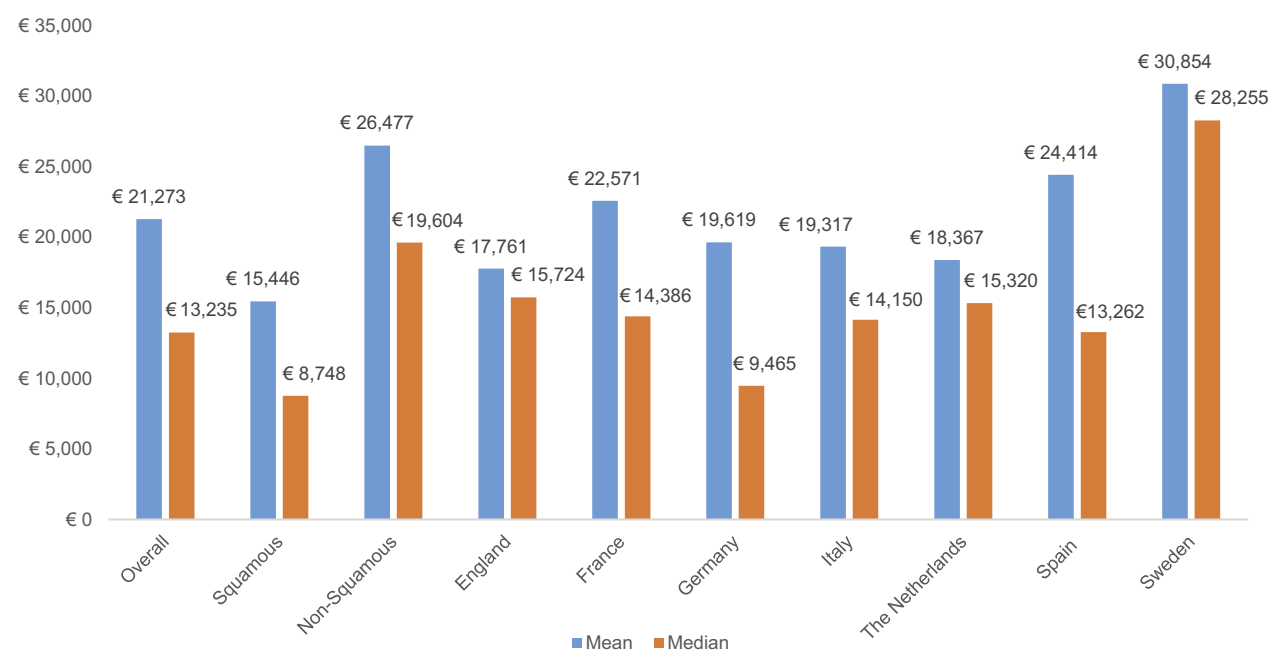

Figure I Total mean per-patient costs related to aNSCLC from aNSCLC diagnosis to most recent visit or death (overall, by histology and by country). 


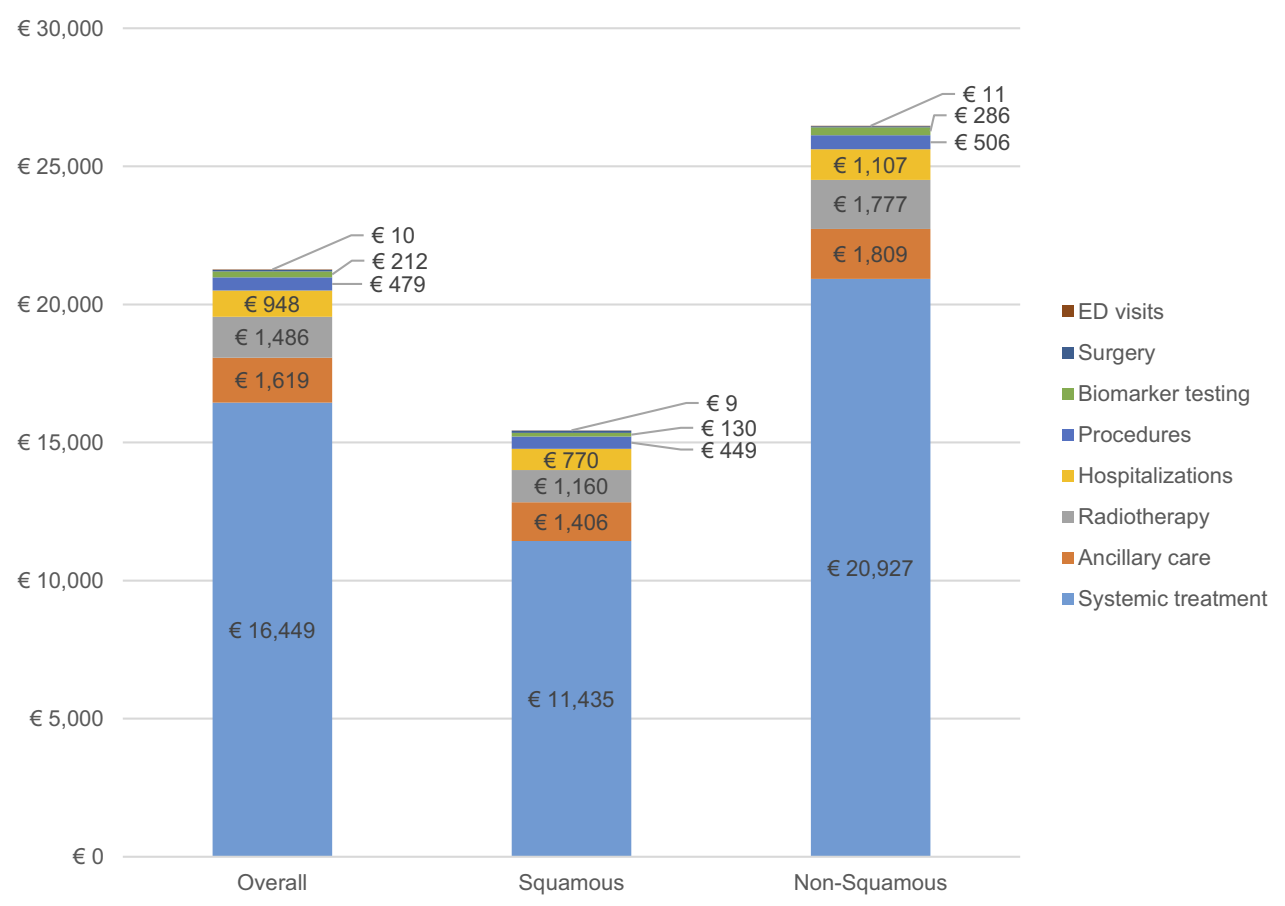

Figure 2 Total per-patient costs related to aNSCLC by cost category (mean values, overall and by histology).

Abbreviation: $E D$, emergency department.

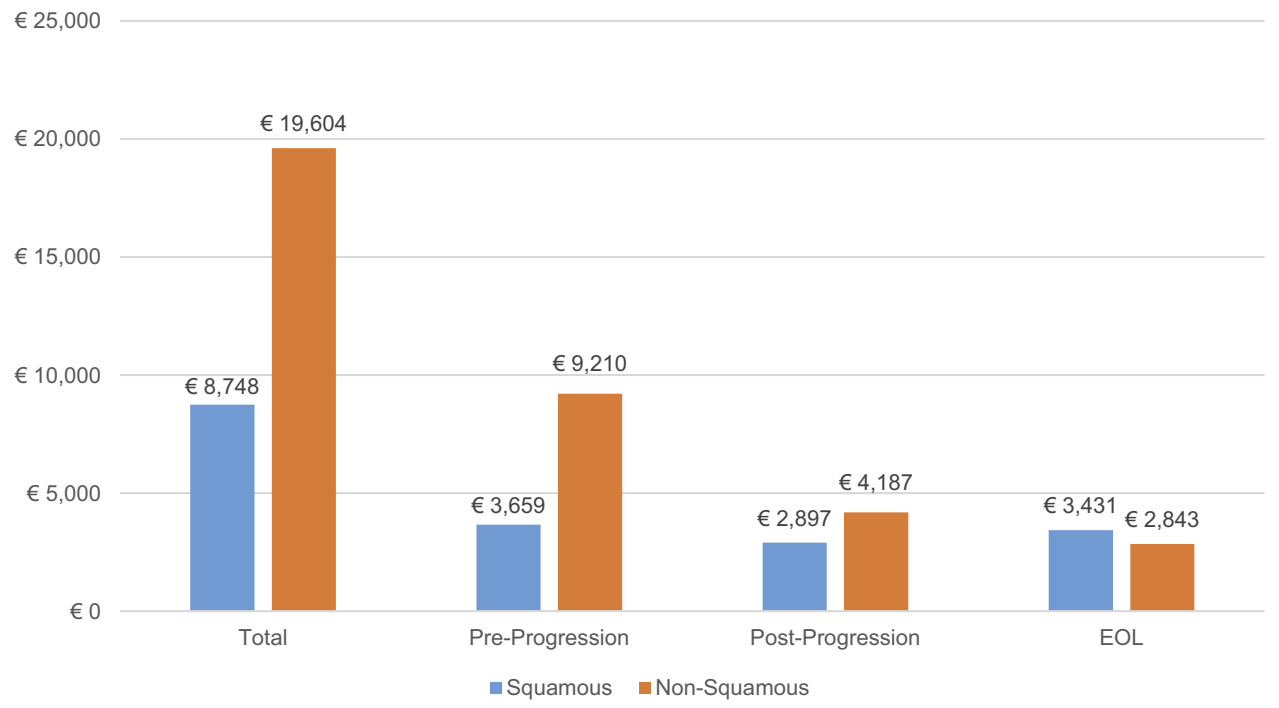

Figure 3 Median costs related to aNSCLC per 2L+ aNSCLC patient by histology and treatment period.

Abbreviations: $2 \mathrm{~L}+$, two or more lines of treatment; $\mathrm{EOL}$, end of life.

Geographical location was also a meaningful driver: relative to Germany, receiving treatment in Spain was an independent significant predictor of higher total costs from aNSCLC diagnosis to most recent visit/death.

When performing the same analysis on costs per patient-month, all variables from the earlier analysis (except age category "65-79 years") and presence of anaplastic lymphoma kinase $(A L K)$ mutation were significant predictors for lower costs (Figure 5). An ECOG PS $\geq 1$ and treatment in France or Italy were additional significant predictors for increased costs.

When restricting the analyses to patients with complete data/who died (sensitivity analysis), the mean per-patient costs related to aNSCLC over the follow-up period were similar to those of the overall cohort, amounting to $€$ 21,728 (SD: $€ 37,615$; median: $€ 13,691$ ). Similarly to the 


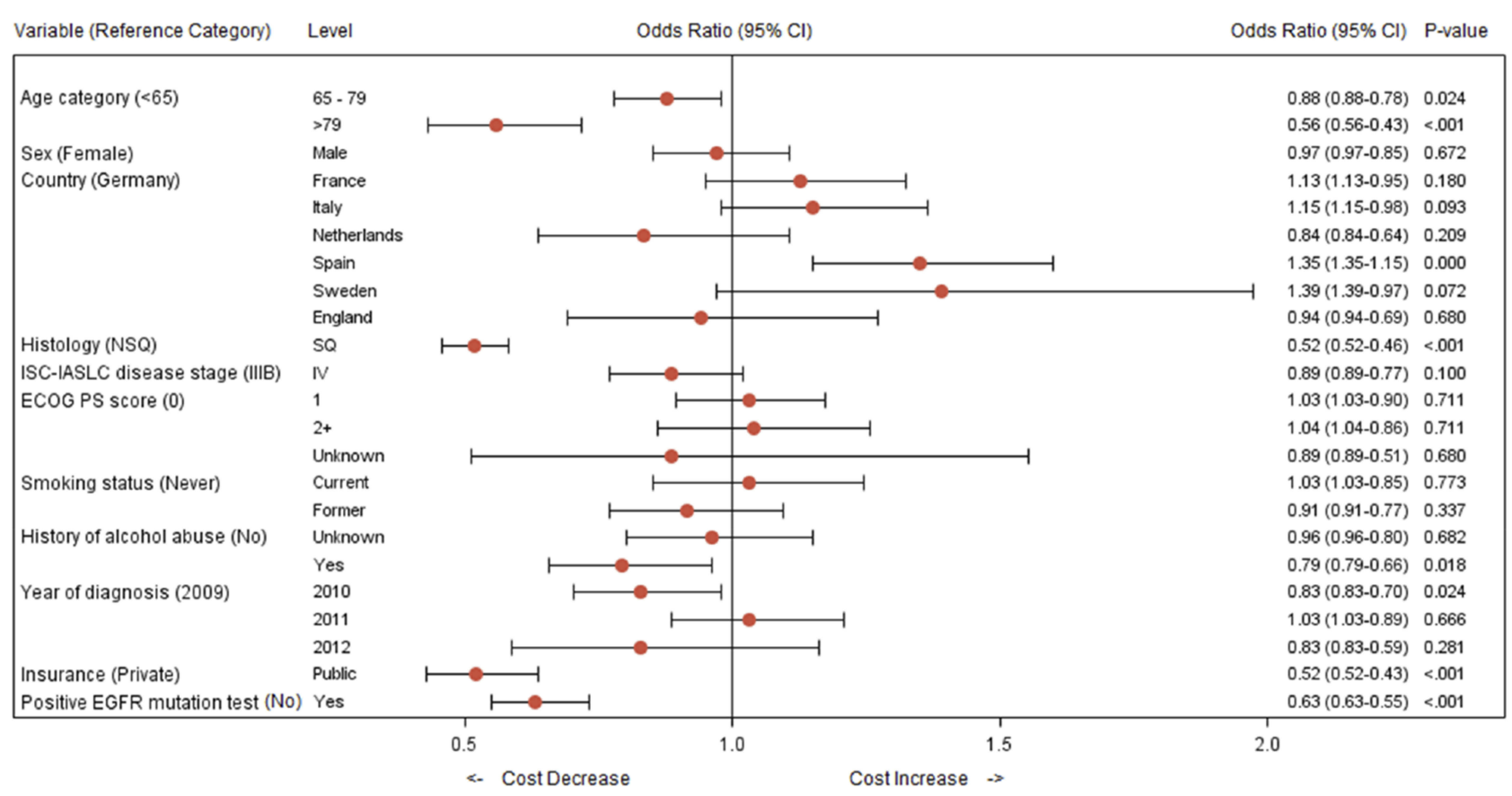

Figure 4 Forest plot of prognostic factors for costs related to aNSCLC from aNSCLC diagnosis to most recent visit/death: total costs per patient. P-value reflects the significance of each explanatory variable, under the assumption that all other variables entered in the model equation are present.

Abbreviations: Cl, confidence interval; ECOG PS, Eastern Cooperative Oncology Group performance status; EGFR, epidermal growth factor receptor gene; ISC-IASLC, International Staging Committee of the International Association for the Study of Lung Cancer; NSQ, non-squamous; SQ, squamous.

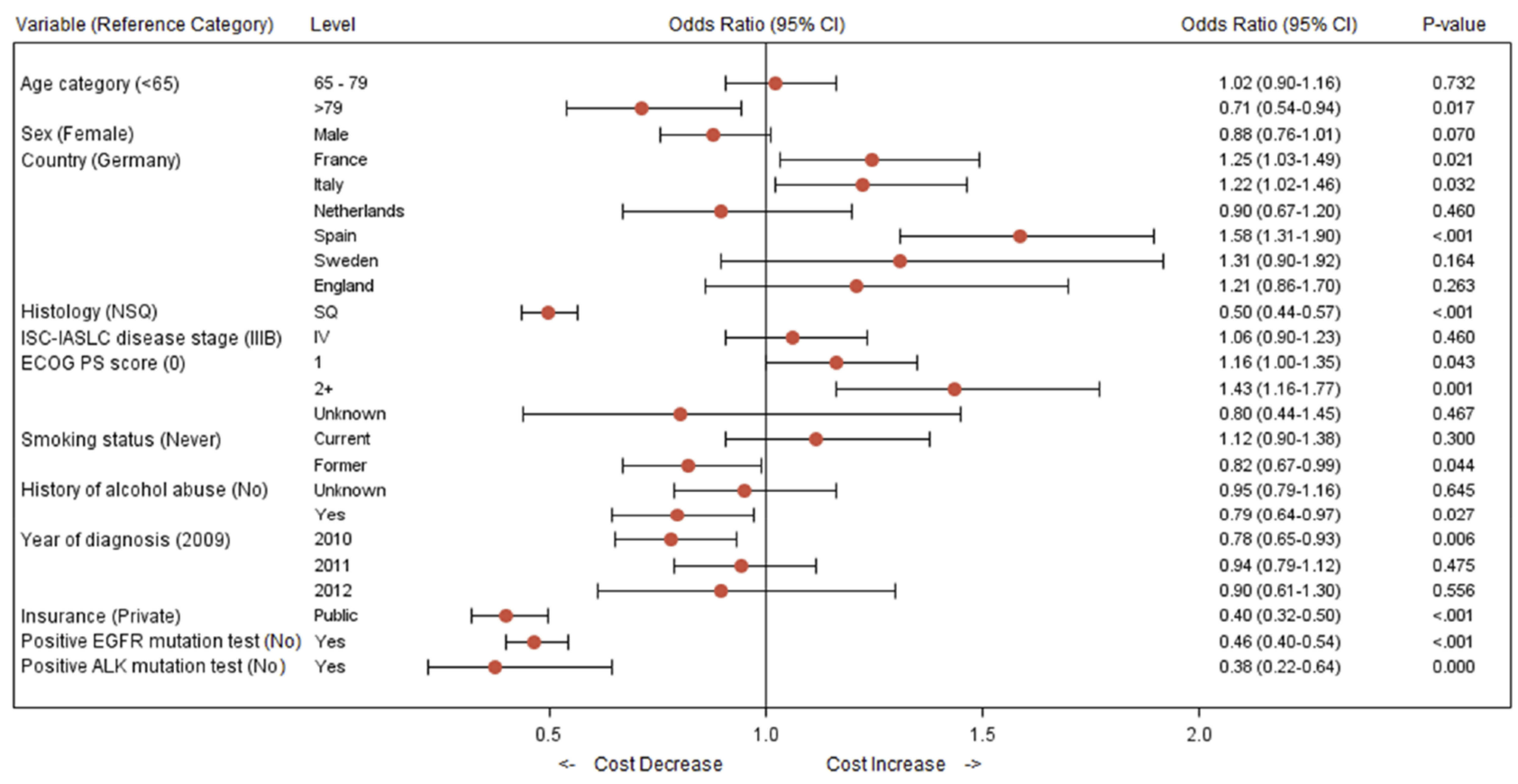

Figure 5 Forest plot of prognostic factors for costs related to aNSCLC from aNSCLC diagnosis to most recent visit/death: total costs per patient-month. P-value test for the significance of each explanatory variable, under the assumption that all other variables entered in the model equation are present.

Abbreviations: ECOG PS, Eastern Cooperative Oncology Group performance status; EGFR, epidermal growth factor receptor gene; ISC-IASLC, International Staging Committee of the International Association for the Study of Lung Cancer; NSQ, non-squamous; SQ, squamous.

overall population, public insurance, presence of $E G F R$ or ALK mutation, SQ histology, advanced age (">79 years"), and being diagnosed in 2010 predicted significantly lower total costs per patient-month. In addition, being a former smoker predicted significantly lower costs, whereas a history of alcohol abuse was not a significant predictor 
$(\mathrm{p}=0.088)$. Treatment in Spain or Italy was a significant predictor for increased costs.

\section{Discussion}

Our retrospective real-world study utilized data from an era prior to the introduction of immunotherapy and reflects the role of systemic anti-cancer medication as a major component of total costs for aNSCLC treatment across Europe among those patients who had progressed to $2 \mathrm{~L}$ treatment. Advanced age ( $\geq 65$ years), SQ histology, public insurance, alcohol abuse, diagnosis in 2010, and presence of EGFR mutation predicted significantly lower total costs per patient. Using average weighted unit costs allowed identification of significant differences across countries that are driven by differences in units of resource use, as opposed to also reflecting the heterogeneity in cross-country costs. Significant cost differences between SQ and NSQ patients were observed in all countries except Spain. To investigate OS as a confounder of the cost driver analysis, total costs per patient-month were also analyzed (Figure 5). All variables remained significant, indicating that they played a role independent of OS in the prediction of costs. The results of the sensitivity analysis demonstrate the robustness of the results.

Costs for systemic anti-cancer medication presented the primary cost driver in this real-world study. This finding may partially be due to the focus on a study population that received two or more lines of treatment, and data that were collected by oncologists/pulmonologists who prescribed systemic anti-cancer drugs. Our findings are, however, in line with costing studies in the Netherlands and Italy. ${ }^{9,10}$ Similar to our study, both studies identified systemic anti-cancer medication as the main cost driver. The total costs of $€ 25,859$ (Italy) ${ }^{9}$ and $€ 17,463$ (The Netherlands) $)^{10}$ are similar to our unweighted results when taking into account their observation times and additional cost items - in the Italian study, ${ }^{9} 15 \%$ of $1 \mathrm{~L}$ costs were due to chemotherapy administration and $9 \%$ due to concomitant medication, which our study did not include in cost analysis.

The impact of disease stage and performance status on costs remains ambiguous. In our study, the additional identification of ECOG $P S \geq 1$ as a predictive factor for higher costs suggests that a more impaired patients' functional status translates into increased costs. This trend was also found in a chart review study in the United States of aNSCLC patients with at least one line of erlotinib or afatinib. ${ }^{11} \mathrm{~A}$ chart review study of hospital-based costs from Brazil, however, suggested an effect of ECOG PS $=2$ leading to lower total costs, which were potentially due to delayed rather than avoided costs. ${ }^{12}$ ISC-IASLC disease stage was not a significant predictor in our study, potentially due to the study being restricted to patients with stage IIIB/IV aNSCLC, but was found a significant predictor in other chart reviews. ${ }^{12,13}$ It may be that ISC-IASLC disease stage impacts indirect costs ${ }^{14}$ or that ECOG PS was not included as an independent variable in these studies. While other studies identified alcohol abuse as a prognostic factor for higher HCRU costs, it was correlated with significantly lower costs (total and per patient-month) in our study. ${ }^{15,16}$ This finding may partly be explained by different extraction patterns, since patients with a history of alcohol abuse in our sample had both shorter OS and less HCRU recorded by the physician. Therefore, the trend is slightly less pronounced when analyzing per patient-month. Since no standardized measure of drinking pattern has been adopted, comparability across studies is limited. ${ }^{17}$

The presence of EGFR mutation was found to be a significant predictor of lower total costs. This result may partly be explained by the high presence of EGFR mutation in our sample. Our study included both a higher percentage of patients who received EGFR biomarker mutation testing (NSQ: $80.2 \%$; SQ: $26.2 \%$ ), and of presence of EGFR mutation (NSQ: $31.1 \%$; SQ: $10.2 \%$ ) than reported in literature, indicating a potential selection bias of patients with better outcomes and lower total costs. ${ }^{18}$ The increasing use of EGFR mutation tests and treatment with tyrosine-kinase inhibitors (TKIs) may also lead to higher treatment costs than found in our sample.

Having private or supplemental insurance was a significant predictor for increased costs (total and per patient-month) in our study. The percentage of patients with unknown insurance status was highest in England (40.4\%), where all residents are insured through the National Health Service. It was thus assumed that patients with reported unknown insurance status had no private or supplemental insurance. This assumption may lead to uncertainty around the parameter estimates. The observed significance of having been diagnosed in the year 2010, as a predictor of lower costs, might be due to potential differences in mortality rates or completeness of data records.

\section{Strengths and Limitations}

A retrospective chart review is an efficient, reliable, and verifiable method of data collection. Medical charts are often the best sources of information for documentation of 
cancer treatments and clinical outcomes. The information collected in the LENS study with regards to detailed aNSCLC characteristics such as histology, ECOG PS, presence of biomarker mutation, reasons for prescribing/discontinuing therapy, and HCRU would not be available in most European registries. However, the study does have limitations. Some parameters which may influence aNSCLC-related costs such as comorbid complications (eg, diabetes COPD, and cardiac conditions) and systemic treatment administration costs were not included in the data collection and could not be accounted for in the analyses. Still, this study fills a gap in existing knowledge of current treatment patterns, clinical outcomes, and HCRU in Europe among patients diagnosed with aNSCLC.

Though efforts were made to ensure physician/patient inclusion criteria were based on random selection, there are risks of selection bias. For example, 96\% of LENS study sites were hospital-based; this is a good representation of real-world treatment, as few NSCLC patients are treated outside of hospital environments, but the study outcomes should not be generalized to patients in other practice settings. Selection bias also may have contributed to the high percentages of patients with EGFR mutations in our study. Validation study results showed that we were more likely to capture patients who were still alive, tending to oversample patients with complete charts. ${ }^{19}$ If mutations are associated with better outcomes, that might explain the higher proportions of patients with mutations in our study. Our validation study also concluded that physicians more often included patients who received mutation testing. ${ }^{19}$ The LENS study may also face a certain bias risk towards patients who were diagnosed late in the inclusion period.

Medical chart abstraction reflects the patterns of country-specific care provision and funding, as known to the participating oncology/pulmonology practices and may capture real-world use of health care resources by patients to a varying degree. In particular, ancillary care reporting conventions vary by country. ${ }^{20}$ The costs are estimated based on average weighted costs per unit at the time of data collection and may not reflect the costs incurred to payers or contemporary costs. Additionally, it should be noted that outpatient physician visits and supportive care were not included in the presented costs, as this information was not collected in sufficient detail to be able to cost.

However, this study's large geographical scope and consistent data collection method facilitate cross-country comparisons within Europe, identification of cost drivers of SQ and NSQ aNSCLC patients, and a detailed assessment of underlying clinical characteristics and treatment patterns of these patients.

\section{Conclusions}

LENS is the first RWE study covering detailed HCRU and cost data among SQ and NSQ aNSCLC 2L+ patients receiving the standard of care prior to the availability of immunotherapy in Europe. In line with results from national European chart review studies, ${ }^{2,9}$ systemic (mostly guideline-consistent) treatment and ancillary care accounted for the majority of total costs. The majority of costs were accrued prior to start of 2L. NSQ patients incurred significantly higher total median costs than SQ patients in all participating countries, and higher mean costs in all countries except Spain, where median costs were slightly higher for SQ patients.

Insurance status, presence of EGFR mutation, SQ histology, age, history of alcohol abuse, and year of diagnosis were significant predictors for lower treatment and HCRU costs per patient-month from aNSCLC diagnosis to most recent visit/death. An ECOG PS $\geq 1$ and country in which treatment was received were significant predictors for higher costs. Other clinically relevant characteristics such as sex, smoking history, and ISC-IASLC disease stage were not significant predictors of total costs. Since little is known about the relationship of HCRU and cost drivers of aNSCLC treatment, ongoing real-word assessments are needed to capture changes in HCRU patterns and costs to provide guidance in the rapidly evolving treatment landscape for aNSCLC.

\section{Acknowledgments}

This study was funded by Bristol-Myers Squibb. Two abstracts of this paper were presented at the ISPOR 20th Annual European Congress as a poster presentation with interim findings. ${ }^{21-23}$

\section{Author Contributions}

Nadine Hertel and Caitlyn Solem directed the study activities. Melinda Manley Daumont, Cynthia Macahilig, and John R Penrod contributed to the study design and interpretation. Linlin Luo performed the analysis for this manuscript. Katharina Verleger supported all project phases, drafted the manuscript and designed the figures and tables. All authors contributed to data analysis, drafting or revising the article, gave final approval of the version to be published, and agree to be accountable for all aspects of the work. 


\section{Disclosure}

Katharina Verleger, Caitlyn Solem, and Linlin Luo are employees of Pharmerit, which was contracted by BristolMyers Squibb to conduct the retrospective chart review study. Cynthia Macahilig is an employee of Medical Data Analytics, which was contracted by Bristol-Myers Squibb to collect and consolidate the data for this study. Nadine Hertel, Melinda Manley Daumont, and John R Penrod are employees of Bristol-Myers Squibb, a global biopharmaceutical company that researches and develops medicines for NSCLC. The authors report no other conflicts of interest in this work.

\section{References}

1. Eurostat. Causes of death statistics - people over 65. In: Eurostat, European Commission. Statistics Explained. 2019:1-15.

2. Malvezzi M, Carioli G, Bertuccio P, et al. European cancer mortality predictions for the year 2018 with focus on colorectal cancer. Ann Oncol. 2018;29:1016-1022. doi:10.1093/annonc/mdy033

3. Jemal A, Bray F, Center MM, Ferlay J, Ward E, Forman D. Global cancer statistics. CA Cancer J Clin. 2011;61(2):69-90. doi:10.3322/caac.v61:2

4. Torre LA, Siegel RL, Ward EM, Jemal A. Global cancer incidence and mortality rates and trends-an update. Cancer Epidemiol Biomarkers Prev. 2016;25(1):16-27. doi:10.1158/1055-9965.EPI-15-0578

5. Allemani C, Matsuda T, Di Carlo V, et al. Global surveillance of trends in cancer survival 2000-14 (CONCORD-3): analysis of individual records for 37513025 patients diagnosed with one of 18 cancers from 322 population-based registries in 71 countries. Lancet. 2018;391(10125):1023-1075. doi:10.1016/S0140-6736(17)33326-3

6. William WN Jr., Lin HY, Lee JJ, Lippman SM, Roth JA, Kim ES. Revisiting stage IIIB and IV non-small cell lung cancer: analysis of the surveillance, epidemiology, and end results data. Chest. 2009;136 (3):701-709. doi:10.1378/chest.08-2968

7. Leidl R, Wacker M, Schwarzkopf L. Better understanding of the health care costs of lung cancer and the implications. Expert Rev Respir Med. 2016;1-3.

8. Novello S, Barlesi F, Califano R, et al. Metastatic non-small-cell lung cancer: ESMO Clinical Practice Guidelines for diagnosis, treatment and follow-up. Ann Oncol. 2016;27(suppl 5):v1-v27. doi:10.1093/ annonc/mdw326

9. Keusters WR, de Weger VA, Hovels A, Schellens JHM, Frederix GWJ. Changing costs of metastatic non small cell lung cancer in the Netherlands. Lung Cancer. 2017;114:56-61. doi:10.1016/j. lungcan.2017.10.005

10. Migliorino MR, Santo A, Romano G, et al. Economic burden of patients affected by non-small cell lung cancer (NSCLC): the LIFE study. J Cancer Res Clin Oncol. 2017;143(5):783-791. doi:10.1007/ s00432-016-2326-x
11. Skinner KE, Fernandes AW, Walker MS, Pavilack M, VanderWalde A. Healthcare costs in patients with advanced non-small cell lung cancer and disease progression during targeted therapy: a real-world observational study. J Med Econ. 2018;21(2):192-200.

12. de Barros Reis C, Knust RE, de Aguiar Pereira CC, Portela MC. Factors associated with non-small cell lung cancer treatment costs in a Brazilian public hospital. BMC Health Serv Res. 2018;18(1):124. doi:10.1186/s12913-018-2933-0

13. Perin J, Zaric B, Dodic JE, et al. The cost of hospital treatment of advanced stage lung cancer patients in a developing South East European country. J Cancer. 2018;9(17):3038-3045. doi:10.7150/ jca. 26278

14. Wood R, Taylor-Stokes G, Lees M, Chirita O. Cost burden associated with advanced Non-Small Cell Lung Cancer (A-NSCLC): impact of disease stage. PCN 87. Value Health. 2017;20:A427. doi:10.1016/j. jval.2017.08.170

15. Fisher MD, Fernandes AW, Olufade TO, Miller PJ, Walker MS, Fenton M. Patient characteristics and costs in recurrent or refractory head and neck cancer: retrospective analysis of a community oncology database. Clin Ther. 2018;40(4):562-573. doi:10.1016/j. clinthera.2018.02.010

16. Paull DE, Updyke GM, Davis CA, Adebonojo SA. Complications and long-term survival for alcoholic patients with resectable lung cancer. $\mathrm{Am}$ J Surg. 2004;188(5):553-559. doi:10.1016/j.amjsurg.2004.07.030

17. Rehm J, Baliunas D, Borges GL, et al. The relation between different dimensions of alcohol consumption and burden of disease: an overview. Addiction. 2010;105(5):817-843. doi:10.1111/j.13600443.2010.02899.x

18. Ryska A, Berzinec P, Brcic L, et al. NSCLC molecular testing in Central and Eastern European countries. BMC Cancer. 2018;18 (1):269. doi:10.1186/s12885-018-4023-4

19. Wilke T, Baeten S, Solem CT, et al. How to design and conduct medical record reviews in oncology. In: ISPOR 19th Annual European Congress, 2016; Vienna.

20. Groeneveld EI, Cassel JB, Bausewein C, et al. Funding models in palliative care: lessons from international experience. Palliat Med. 2017;31(4):296-305. doi:10.1177/0269216316689015

21. Solem C, Penrod JR, Lees M, et al. Cost drivers of lung cancer care: results from a retrospective chart review of pretreated advanced NSCLC patients in Europe. In: International Society for Pharmacoeconomics and Outcomes Research Conference, 2017; Glasgow. doi:10.1016/j. jval.2017.08.194

22. Solem C, Penrod JR, Lees M, et al. Quantification of real-world health care resource utilisation and related costs among patients who received at least two lines of treatment for advanced NSCLC in England, the Netherlands, and Sweden. In: International Society for Pharmacoeconomics and Outcomes Research Conference, 2017; Glasgow.

23. Solem CT, Penrod JR, Lees M, et al. Resource utilization among advanced squamous and non-squamous non-small cell lung cancer patients receiving second-line treatment in France, Germany, Italy, and Spain: results of a retrospective medical chart review. Value Health. 2015;18(7):A450. doi:10.1016/j.jval.2015.09.1133
ClinicoEconomics and Outcomes Research

\section{Publish your work in this journal}

ClinicoEconomics and Outcomes Research is an international, peerreviewed open-access journal focusing on Health Technology Assessment, Pharmacoeconomics and Outcomes Research in the areas of diagnosis, medical devices, and clinical, surgical and pharmacological intervention. The economic impact of health policy and health systems organization also constitute important areas of coverage. The manuscript management system is completely online and includes a very quick and fair peer-review system, which is all easy to use. Visit http://www.dovepress.com/testimonials.php to read real quotes from published authors. 